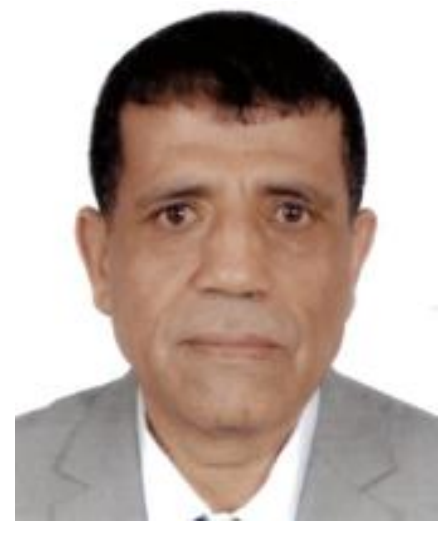

\title{
Photovoltaic: Perovskite fever: Is Perovskite the future of solar cells?
}

\author{
Professor Aziz Naamane \\ LIS UMR CNRS 7020 Aix Marseille University, CNRS SASV and HyRES Lab \\ Marseille, France \\ Email: aziz.naamane@lis-lab.fr
}

Perovskites, this somewhat "barbaric" term derived from the name of a 19th century Russian mineralogist, Lev Perovski, is not yet known to the public. But in labs around the world researchers are feverishly active around this family of crystals that promise to revolutionize the conversion of solar energy into electricity.

Originally the perovskite is a crystal composed of calcium, titanium and oxygen. However, the term is now used to refer to other chemical compounds with identical structures. About ten years ago these materials, then used to make certain electronic components, suddenly emerged as potential candidates for the replacement of expensive silicon cells in solar panels. The hope was mainly based on the cost of perovskite cells, which are significantly lower than those made with silicon crystals and which currently equip most photovoltaic installations

\section{Perovskite at a glance}

- Perovskite solar cells are cheap, easy to implement and efficient, but their operation still lacks stability.

- The latest developments in research tend to move closer to the goal: solar cells with long-term high performance.

- To be successful, the new technology must be compatible with existing industrial manufacturing processes.

- A new technology is in the starting blocks: solar cells with perovskites. This generic term refers to new materials whose crystalline structure is similar to that of the natural material called perovskite.

- The solar cells with perovskites have undergone a flash development. Over the past decade, their output has literally exploded from just $3 \%$ to over $20 \%$. Perovskites have remarkable properties: they absorb light particularly efficiently and evacuate the current produced well. In addition, they are both cheap and easy to manufacture and process. However, perovskite-based solar cells also have a significant drawback: they do not yet function consistently enough and are not durable enough for large-scale use. Researchers from the ETH Lausanne and the University of Freiburg are working to improve the stability and performance of this promising technology.

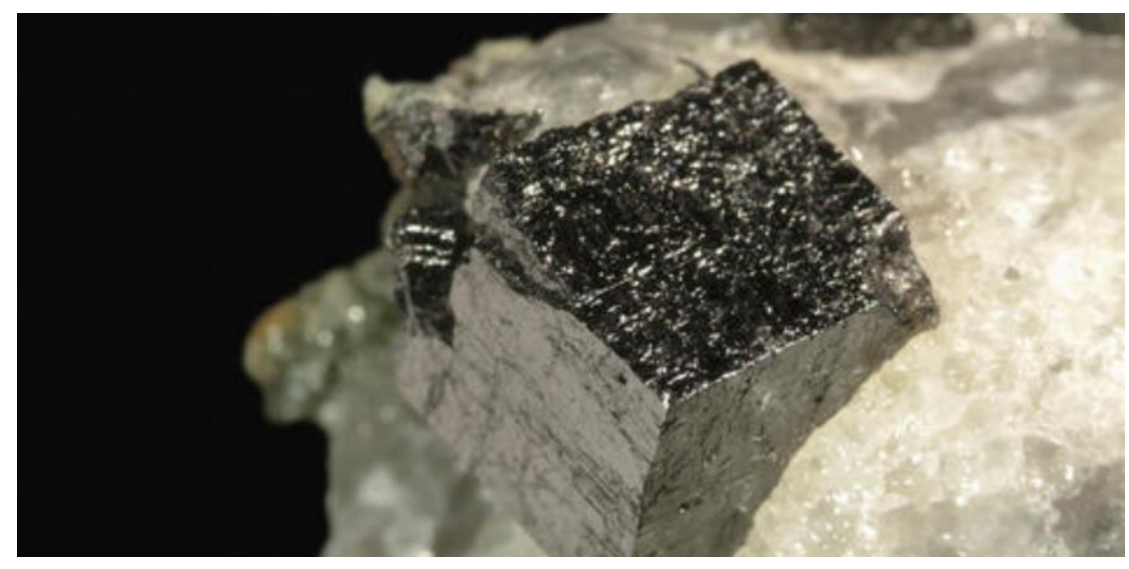

Perovskite rock in the Urals massif, Russia.

The discovery of the photovoltaic qualities of perovskites dates back only to 2012 . 


\section{Conclusions:}

In short, it would be tedious to describe all the research currently underway, but be aware that the results are there: lately not several weeks go by without a team proudly announcing that they have made progress towards the development of a photovoltaic technology based on perovkites, much cheaper than the current silicon cells and whose lifespan and efficiency are equivalent. Theoretically, the yield of perovskite-based panels could even be as high as $30 \%$, but we are not there yet.

Another area of research concerns the manufacturing technologies of perovskite-based cells. Scientists are working on the possibility of placing thin-film materials on a flexible PET medium [1] using a process similar to inkjet printers. The solar module that we get is almost transparent, very flexible and offers a wide range of possibilities. For example, it could be integrated into building glazing or in complex shapes such as vehicle bodies. Some even plan to make clothes out of them.

The final issue is cost and marketing. According to media estimates, the price of perovskite-based solar modules is 4 to 5 times lower than that of current photovoltaic panels.

If all these hopes and promises come true, we may well be on the cusp of a solar revolution that would definitively sound the death knell for fossil fuels and nuclear power.

Shall we agree that Perovskites: these crystals will revolutionize solar energy? this question remains open.

[1] PET - polyethylene terephthalate: it is the plastic used to make bottles containing soft drinks

\section{References}

[1] Frohna, Samuel D. Stranks, in Handbook of Organic Materials for Electronic and Photonic Devices (Second Edition), 2019.

[2] NREL's 'Best Research-Cell Efficiencies'. Available at: https://www.nrel.gov/pv/assets/pdfs/pv-efficiencies07-17-2018.pdf. (Accessed: 14th December 2018)

\section{About Professor Aziz Naamane}

Dr Abdelaziz Naamane obtained a M.Sc. in electronics, power electronics, and automatic control from the Université de Provence and a Ph.D. in electronics and physics from Aix Marseille University. He is currently a Professor at Aix Marseille Université and a senior researcher at Laboratoire d'Informatique \& Systèmes (LIS UMR 7020 CNRS)

CURRENT INTEREST: Modelling, simulation and control of complex hybrid systems. Control-oriented modelling of nonlinear dynamical systems. Energy-based modelling (Bond Graphs, Euler-Lagrange, port-Hamiltonian methods) and control of nonlinear physical systems. Applications of nonlinear techniques to modelling, analysis and control of electrical machines, power electronics drives, and general mechatronic systems, including robotics. Energy management and control in micro smart grids. 